\title{
Numerical Investigation of Multi-Nozzle Propulsive Deceleration Jets for Mars Entry Aeroshells
}

\author{
Hicham Alkandry* and Iain D. Boyd ${ }^{\dagger}$ \\ Department of Aerospace Engineering, University of Michigan, Ann Arbor, MI, 48109 \\ Joshua R. Codoni ${ }^{\ddagger}$, Erin M. Reed ${ }^{\S}$, and James C. McDaniel ${ }^{\llbracket}$ \\ Department of Mechanical and Aerospace Engineering, University of Virginia, Charlottesville, VA, 22903
}

\begin{abstract}
The effects of peripheral propulsive decelerator (PD) jets on the deceleration performance of Mars-entry aeroshells are examined using the computational fluid dynamics code LeMANS and the planar laser-induced iodine fluorescence experimental technique. The study uses an aeroshell based on the Mars Science Laboratory with four peripheral jets in Mach 12 flow of $I_{2}$-seeded $N_{2}$ gas. Sonic and supersonic peripheral jets are considered in this study. The results show that the peripheral PD jets increase the standoff distance and change the profile of the bow shock around the aeroshell. The results also show that PD jets obstruct the flow around the aeroshell, which creates a low pressure region on the surface behind the jets. The level of obstruction increases with thrust, which expands the low pressure region. As a result, the drag on the aeroshell is inversely proportional to the thrust. The PD jet Mach number, however, has a small effect on the deceleration performance, with a maximum difference of less than $2.5 \%$ in the axial force between sonic and supersonic peripheral jets. The study also compares the deceleration performance of peripheral PD jets to previously published results for central PD jets. The results show that the peripheral jets provide 3 to 8 times larger drag than central jets due to higher surface pressures. Finally, qualitative comparisons between the numerical results and experimental data show overall good agreement in the bow shock standoff distance and profile.
\end{abstract}

\section{Nomenclature}

$C_{A} \quad$ Axial Force Coefficient

$C_{D} \quad$ Drag Coefficient

$C_{P} \quad$ Pressure Coefficient

$C_{T} \quad$ Thrust Coefficient

$F_{D} \quad$ Drag Force $[\mathrm{N}]$

$F_{T} \quad$ Thrust Force [N]

Kn Knudsen Number

$M \quad$ Mach Number

$\dot{m} \quad$ Mass Flow Rate $[\mathrm{kg} / \mathrm{s}]$

$P \quad$ Pressure $[\mathrm{Pa}]$

$q \quad$ Dynamic Pressure [Pa]

Re Reynolds Number

$S \quad$ Aeroshell Frontal Area $\left[\mathrm{m}^{2}\right]$

$U \quad$ Velocity $[\mathrm{m} / \mathrm{s}]$

$X \quad$ Mole Fraction

* Graduate Student, Student Member AIAA.

$\dagger$ James E. Knott Professor, Fellow AIAA.

${ }^{\ddagger}$ Graduate Student, Student Member AIAA.

$\S$ Graduate Student, Student Member AIAA.

IProfessor, Associate Fellow AIAA. 
$\gamma \quad$ Ratio of Specific Heats

$\rho \quad$ Density $\left[\mathrm{kg} / \mathrm{m}^{3}\right]$

subscripts

0 Total (Stagnation) Conditions

jet PD Jet Conditions

ref Reference Freestream Conditions

\section{Introduction}

Luture Mars missions, including possible human-exploration missions, will have landed masses larger by $\mathrm{H}$ as much as two orders of magnitude and landing site elevations much higher than previous missions. The conventional aerodynamic decelerators, such as the disk-gap-band parachute and the $70^{\circ}$ sphere-cone, that have been used on all Mars missions to date, as well as the upcoming Mars Science Laboratory (MSL), may be inadequate because they cannot be simply extended to these future mission requirements. ${ }^{1}$ Therefore, propulsive deceleration is currently being investigated as an alternative method for slowing future Mars-entry aeroshells during atmospheric descent. Propulsive decelerator (PD) jets work by directing thrust from an engine into the incoming freestream flow. The PD jets can be arranged in either a central configuration, with only a single jet located at the center of the aeroshell, or in a peripheral configuration, with several jets located around the center of the aeroshell. Experimental work, mainly from the 1960s and 1970s, has shown

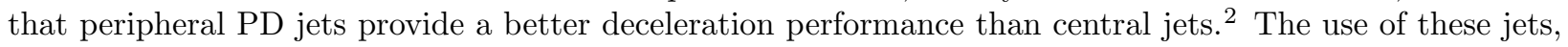
however, generates complex fluid interactions between the jets, the freestream, and the aeroshell that are still not well understood.

Currently, there are several ongoing studies that aim to understand the fluid interactions of PD jets using both experimental and numerical methods. ${ }^{3-5}$ These studies focus on supersonic retro-propulsion (SRP), which involves using propulsive deceleration in a supersonic freestream. However, several different entry, descent, and landing (EDL) architectures involving propulsive deceleration have been proposed, ${ }^{6}$ including an all-propulsive design with PD jets firing into an incoming hypersonic freestream. Therefore, this study focuses on using PD jets in a hypersonic freestream and expands on previous analysis, which focused on central PD jets, ${ }^{7,8}$ to peripheral jets. The paper will first describe experimental and numerical approaches that are used to understand the interactions that are generated by the peripheral PD jets. The paper will then present numerical results in two parts. The first section will examine the effects of the jet Mach number on the flowfield and aerodynamic properties of a Mars-entry aeroshell with peripheral PD jets. The second section of the results will compare and analyze the deceleration performance of peripheral and central PD jets. In the last section of paper, qualitative comparisons between the numerical results and experimental data will be carried out in order to assess the accuracy of the computational method.

\section{Technical Approach}

\section{A. Experimental Technique}

Experimental results are obtained using the planar laser-induced iodine fluorescence (PLIIF) technique in a hypersonic wind tunnel facility at the University of Virginia. The PLIIF technique is a non-intrusive, spatially-resolved, time-averaged optical method for obtaining measurements in hypersonic, rarefied flows. The technique has been used for both qualitative and quantitative measurements. ${ }^{9-11}$ PLIIF involves seeding iodine into a flowfield and exciting the molecules to a higher energy with an argon ion laser. The laser beam is turned into a thin laser sheet and passed through the flowfield of interest. The resulting fluorescence is imaged at $90^{\circ}$ using a cooled scientific-grade charge-coupled device (CCD) camera. Measurements of the absorption spectrum are made as the laser is tuned in frequency. By fitting the measured absorption spectra at every point in the flowfield, the velocity, pressure, density, and temperature can be deduced. The technique provides qualitative flow visualization images when the laser is operated in the broadband mode, in which the laser gain profile is much wider than the iodine absorption linewidth. The results to be presented herein have been taken with this approach.

The hypersonic flow facility at the University of Virginia is capable of providing Mach numbers and 
Knudsen numbers up to 16 and 1, respectively. Hypersonic flow from an under-expanded jet is produced by the expansion of iodine-seeded nitrogen gas through a thin circular orifice of diameter $D=2 \mathrm{~mm}$ into a continuously evacuated vacuum chamber. The stagnation pressure and temperature in the wind tunnel are $1.8 \mathrm{~atm}$ and $300 \mathrm{~K}$, respectively. Figure 1(a) presents a schematic of the experimental setup in the hypersonic flow facility. Figure 1(b) shows calculated Mach number and Knudsen number $(K n)$ variations inside the freejet facility. ${ }^{9}$ These contours show the barrel shock that develops at the entrance of the test section and terminates at the Mach disk. Models are placed in the under-expanded jet core for testing at hypersonic conditions. The freestream Mach number and flow properties can be changed by adjusting the distance of the test model to the orifice.

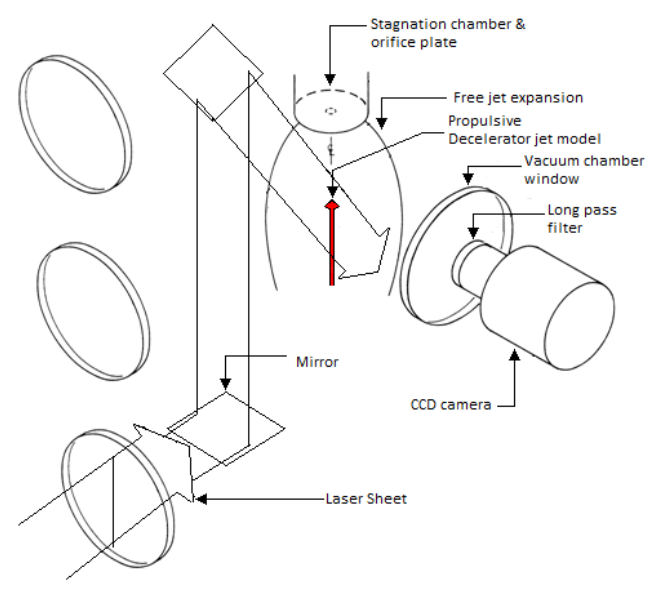

(a) Schematic of the experimental setup

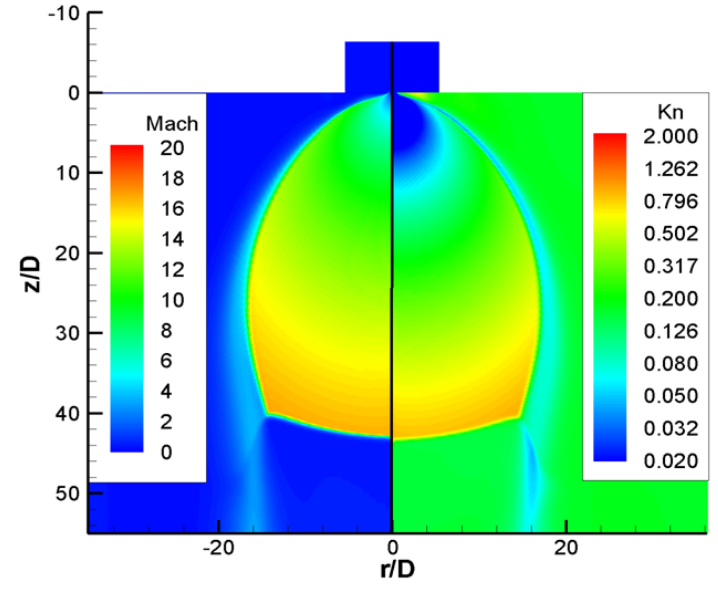

(b) Calculated Mach number and Knudsen number contours in the test section

Figure 1. Experimental facility.

\section{B. Numerical Method}

Numerical simulations are performed using the computational fluid dynamics (CFD) code LeMANS, developed at the University of Michigan for simulating hypersonic reacting flows. ${ }^{12,13}$ This general purpose, three-dimensional, parallel code solves the laminar Navier-Stokes equations on unstructured computational grids including thermo-chemical nonequilibrium effects with second-order spatial accuracy. The flow is modeled assuming that the continuum approximation is valid. Furthermore, for this work, it is assumed that the translational and rotational energy modes of all species can be described by two different temperatures $T_{\text {tra }}$ and $T_{r o t},{ }^{14}$ respectively, while the vibrational energy and electronic energy of all species are frozen at the stagnation value (i.e. $300 \mathrm{~K}$ ). In LeMANS, the mixture transport properties can be computed using several options. In this study, Wilke's semi-empirical mixing rule ${ }^{15}$ is used with species viscosities calculated using Blottner's model ${ }^{16}$ and species thermal conductivities determined using Eucken's relation. ${ }^{17}$

The finite-volume method applied to unstructured grids is used to solve the set of partial differential equations. LeMANS can simulate two-dimensional and axisymmetric flows using any mixture of quadrilateral and triangular mesh cells, and three-dimensional flows using any mixture of hexahedra, tetrahedra, prisms, and pyramids. A modified Steger-Warming Flux Vector Splitting scheme is used to discretize the inviscid fluxes across cell faces, which is less dissipative and produces better results in boundary layers compared to the original scheme. The viscous terms are computed using cell-centered and nodal values. In this study, time integration is performed using a point implicit method. LeMANS is parallelized using METIS ${ }^{18}$ to partition the computational mesh, and MPI to communicate the necessary information between processors. 


\section{Numerical Setup}

Figure 2 presents the aeroshell geometry with sonic and supersonic peripheral PD jets. Due to symmetry in the flowfield, the computational domain consists of only one quarter of the aeroshell (dark shaded sections in Figure 2) in order to reduce cost and complexity. The computational geometry also includes a "sting" attached to the backshell, which is used in the experiments to hold the model in the test section and supply the flow to the PD jet. The aeroshell diameter for both PD configurations is $10 \mathrm{~mm}$, which is approximately $0.22 \%$ of the MSL aeroshell diameter. The PD nozzles are located halfway between the forebody center (i.e. stagnation point) and the aeroshell shoulder and are directed parallel to the main freestream flow. The diameter of the PD nozzle-exit is $0.5 \mathrm{~mm}$ and $0.9 \mathrm{~mm}$ for the sonic and supersonic jets, respectively. The computational grids are adapted by hand from previous simulations in order to ensure that the upstream boundary is aligned with the bow shock, as well as adequate mesh resolution. The grids use hexahedral cells with clustering near the wall and in the vicinity of the PD jet in front of the aeroshell. The grid size varies from about 4 million to approximately 5 million cells.

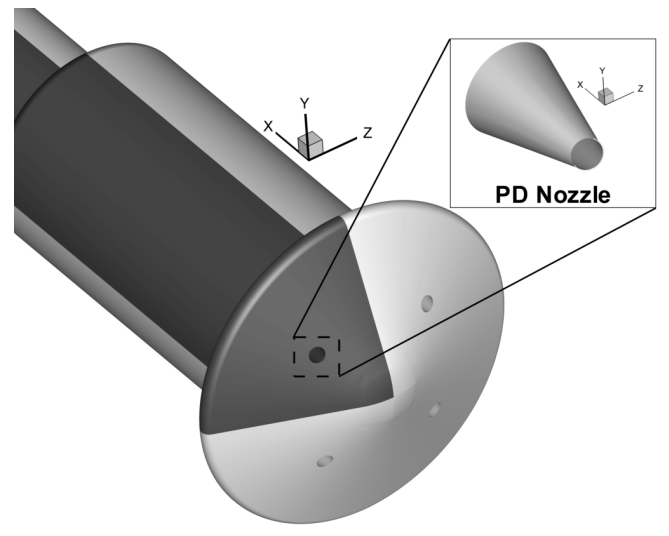

(a) Sonic PD jet

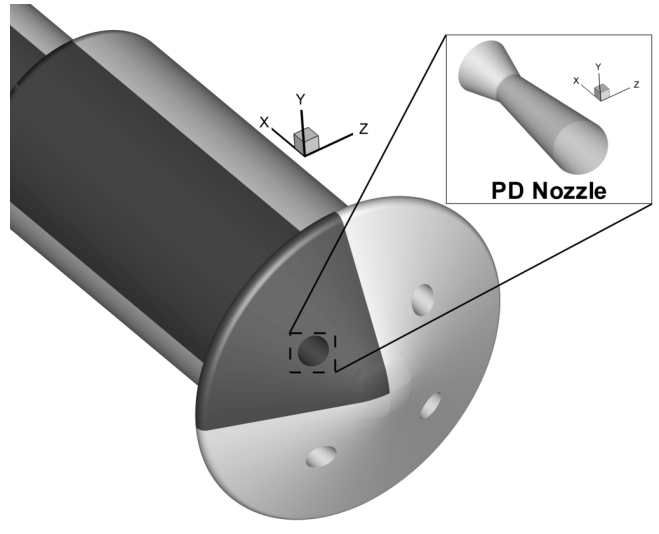

(b) Supersonic PD jet

Figure 2. Aeroshell geometry with sonic and supersonic peripheral PD jet (dark shade: geometry used in the computational simulations).

$\mathrm{I}_{2}$-seeded $\mathrm{N}_{2}$ gas is used in the numerical simulations with a seeding ratio of $200 \mathrm{ppm}$ for both the freestream and the PD jet flows in order to accurately model the experimental conditions. Radially nonuniform conditions based on the freejet relations of Ashkenas and Sherman ${ }^{19}$ are also used as flow conditions input to LeMANS at the upstream boundary. A previous study showed that these nonuniform freestream conditions widen the bow shock around the aeroshell and decrease the drag coefficient by $6.4 \%$ compared to the uniform conditions. ${ }^{20}$ The Mach number at a distance $z$ away from the orifice along the centerline of the freejet is given by Equation 1

$$
M=A\left(\frac{z-z_{0}}{D}\right)^{\gamma-1}-\frac{1}{2}\left(\frac{\gamma+1}{\gamma-1}\right)\left[A\left(\frac{z-z_{0}}{D}\right)^{\gamma-1}\right]^{-1}
$$

where $D$ is the diameter of the freejet orifice, and $A$ and $z_{0} / D$ are constants determined for values of the ratio of specific heats $\gamma$, and are equal to 3.65 and 0.40 , respectively, for $\gamma=1.4$. All other fluid properties along the freejet axis can be computed using the Mach number defined in Equation 1, the stagnation conditions in the wind tunnel and the isentropic relations. The density distribution at a fixed distance from the orifice exit is a function of the streamline angle $\theta$ with respect to the freejet axis as shown in Equation 2

$$
\frac{\rho(\theta)}{\rho(0)}=\cos ^{2}\left(\frac{\pi \theta}{2 \Phi}\right)
$$

where $\Phi$ is also a constant determined for each value of $\gamma$, and is equal to 1.662 for $\gamma=1.4$. For this study, a reference freestream Mach number of 12 is used in order to minimize the interaction of the bow shock around the aeroshell and the barrel shock created in the test section in the experiments. As a result, it is not necessary to model the entire test section of the wind tunnel in the numerical simulations, which 
dramatically cuts down on the computational cost and complexity. The freestream rotational temperature is assumed to be equal to the translational temperature. The aeroshell wall is assumed to be at a constant temperature of $300 \mathrm{~K}$. Figure 3, modified from McDaniel et al. ${ }^{9}$ shows a to-scale plot of the location of the aeroshell model with respect to the freejet orifice and velocity streamlines for the Ashkenas and Sherman boundary conditions. A set of reference freestream conditions is obtained using isentropic relations for a reference freestream Mach number of 12. These reference conditions are presented in Table 1 and are used to compute non-dimensional quantities, such as the drag coefficient. The low Reynolds number based on these reference conditions and the aeroshell diameter indicates that the freestream flow is laminar.

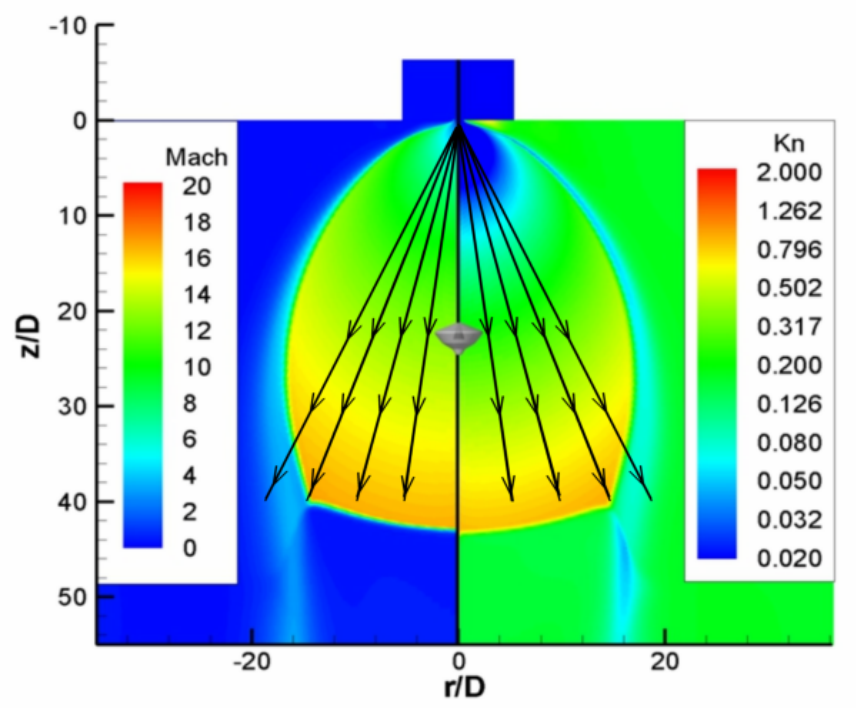

Table 1. Reference freestream conditions.

\begin{tabular}{lc}
\hline \hline Parameter & Value \\
\hline$M_{\text {ref }}$ & 12 \\
$\rho_{\text {ref }}, \mathrm{kg} / \mathrm{m}^{3}$ & $4 \times 10^{-4}$ \\
$U_{\text {ref }}, \mathrm{m} / \mathrm{s}$ & 776 \\
$R e_{\text {ref }}$ & 1200 \\
\hline \hline
\end{tabular}

Figure 3. Ashkenas and Sherman boundary conditions and the position of the model in the test section.

The boundary conditions for the PD jet are computed such that either sonic $\left(M_{\text {jet }}=1.00\right)$ or supersonic $\left(M_{j e t}=2.66\right)$ conditions are obtained at the nozzle-exit. These conditions are non-dimensionalized using the thrust coefficient given in Equation 3,

$$
C_{T}=\frac{F_{T}}{q_{r e f} \cdot S}=\left(2 \frac{q_{j e t}}{q_{\text {ref }}}+\frac{P_{j e t}}{q_{r e f}}-\frac{P_{a m b}}{q_{r e f}}\right)\left(\frac{A_{j e t}}{S}\right)
$$

where $P_{a m b}$ is the post-bow shock static pressure and $A_{\text {jet }}$ is the area of the PD nozzle-exit. Tables 2 and 3 present the total pressure ratio, the ideal mass flow rate, and the Reynolds number based on the nozzle-exit conditions for the sonic and supersonic PD jet configurations, respectively. These conditions are specified for each PD jet and are obtained such that the total thrust coefficient of all four peripheral jets is equal to the value shown in the tables. The flow from the PD nozzle is assumed laminar since the jet Reynolds number for all the cases is less than $10^{4} .{ }^{21}$

Table 2. Sonic PD jet boundary conditions.

\begin{tabular}{cccc}
\hline \hline$C_{T}$ & $P_{0, j e t} / P_{0}$ & $\dot{m}, \mathrm{~kg} / \mathrm{s}\left(\times 10^{-6}\right)$ & $R e$ \\
\hline 0.5 & 0.03 & 2.1 & 370 \\
1.0 & 0.06 & 4.1 & 720 \\
1.5 & 0.08 & 6.1 & 1050 \\
2.0 & 0.11 & 8.2 & 1450 \\
2.5 & 0.14 & 10.2 & 1800 \\
\hline \hline
\end{tabular}

Table 3. Supersonic PD jet boundary conditions.

\begin{tabular}{cccc}
\hline \hline$C_{T}$ & $P_{0, j e t} / P_{0}$ & $\dot{m}, \mathrm{~kg} / \mathrm{s}\left(\times 10^{-6}\right)$ & $R e$ \\
\hline 0.5 & 0.02 & 2.1 & 280 \\
1.0 & 0.05 & 3.9 & 530 \\
1.5 & 0.07 & 5.8 & 780 \\
2.0 & 0.09 & 7.7 & 1040 \\
2.5 & 0.11 & 9.6 & 1300 \\
\hline \hline
\end{tabular}




\section{Results}

The goal of this study is to understand the effects of peripheral PD jets on the deceleration performance of a Mars entry aeroshell. The numerical results are also used in qualitative comparisons with experimental data to assess the accuracy of the computational method. The surface and aerodynamic properties are presented as non-dimensional pressure, drag, and axial force coefficients, given in Equations 4, 5, and 6, respectively,

$$
\begin{gathered}
C_{P}=\frac{P}{(1 / 2) \rho_{r e f} U_{r e f}^{2}} \\
C_{D}=\frac{F_{D}}{(1 / 2) \rho_{r e f} U_{r e f}^{2} S} \\
C_{A}=C_{D}+C_{T}
\end{gathered}
$$

Figure 4 shows Mach number contours for the no-jet and the sonic peripheral PD jet with $C_{T}$ of 1.5 configurations. The no-jet case uses a clean geometry without the nozzle cavity. The bottom half is a reflection of the top half since only one quarter of the geometry is used in the numerical simulations. The flow around the aeroshell without the PD jet is characterized by a bow shock that develops around the aeroshell and a series of expansion and compression waves that occur along the aeroshell aftbody and sting. Figure 4(b) shows that the peripheral PD jet expands from sonic conditions at the nozzle exit to supersonic and hypersonic conditions in front of the aeroshell. The jet decelerates to subsonic conditions through a jet shock before mixing with the freestream flow. The peripheral PD jet interacts with the bow shock by pushing the section close to the jet plume upstream and changing the shock profile to a "saddle" shape. As a result, some of the freestream flow between the peripheral PD jets passes through a nearly normal shock. Between two adjacent PD jets, the level of interaction with the bow shock diminishes, as can be seen in Figure 4(c). Although the bow shock standoff distance is still greater than for the no-jet case, the bow shock recovers its shape between the peripheral jets and the flowfield structure closely resembles the one without the PD jets.

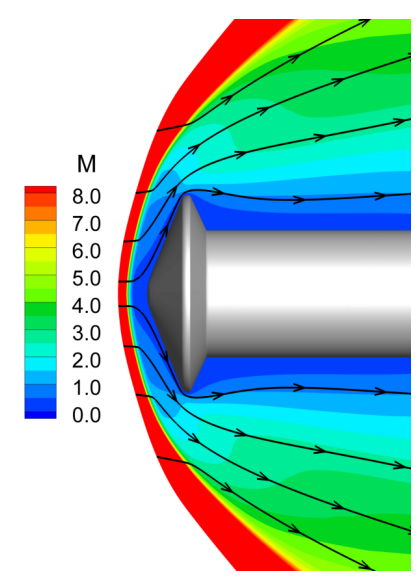

(a) No jet

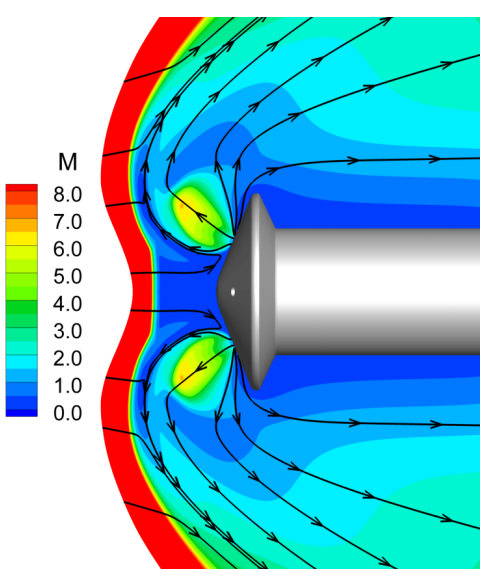

(b) Peripheral PD jet (cut-plane along the (c) Peripheral PD jet (cut-plane between $\mathrm{PD}$ jet axis)

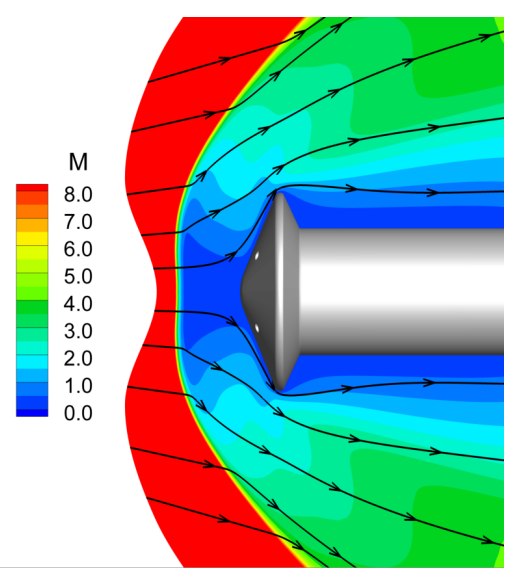

2 adjacent $\mathrm{PD}$ jets)

Figure 4. Mach number contours for the no-jet and sonic peripheral PD jet $\left(C_{T}=1.5\right)$ configurations.

\section{A. Effects of Peripheral PD Jet Mach Number}

Figure 5 presents Mach number contours and velocity streamlines for sonic (top) and supersonic (bottom) peripheral PD jets at various thrust coefficients. The contours show that the supersonic PD jet is narrower and penetrates farther than the sonic jet, particularly for the larger thrust coefficients. As a result, the interaction between the PD jet and the bow shock is stronger for the supersonic configuration. The bow shock standoff distance and the shock curvature near the PD jet are also larger for the supersonic jets. This causes the normal portion of the bow shock between the PD jets upstream of the aeroshell nose to decrease in size. 


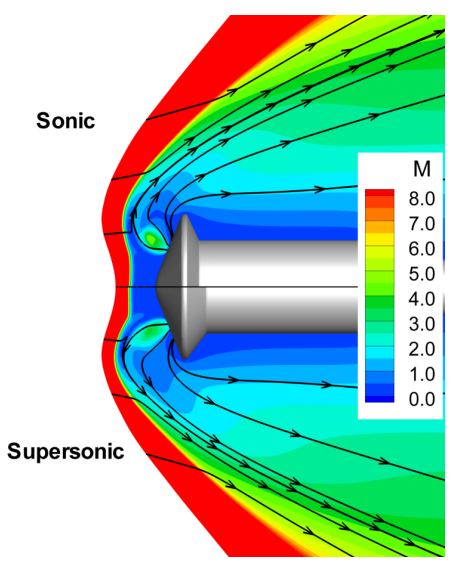

(a) $C_{T}=0.5$

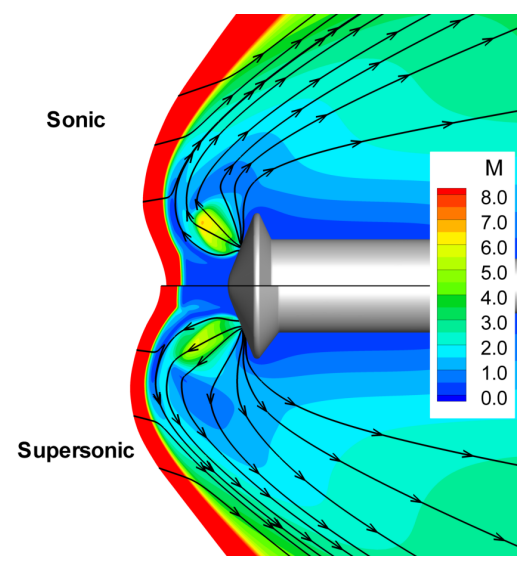

(b) $C_{T}=1.5$

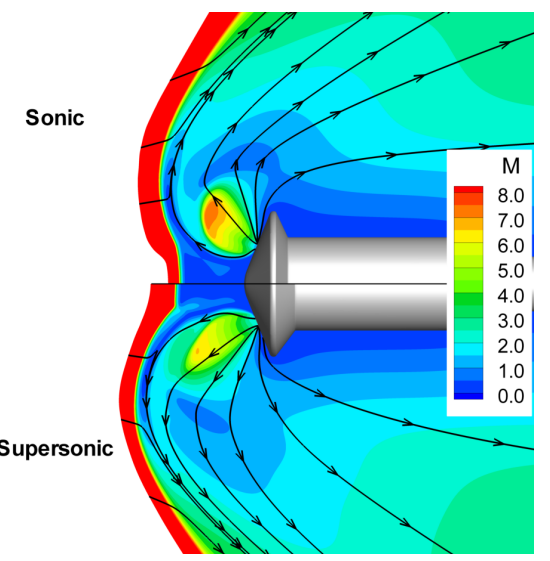

(c) $C_{T}=2.5$

Figure 5. Mach number contours for sonic (top) and supersonic (bottom) peripheral PD jets.

The drag and axial force coefficients, calculated using Equations 5 and 6, for the sonic and supersonic peripheral PD jets are presented in Figure 6 . The figure shows very similar trends and overall close values of drag and axial force coefficients for the sonic and supersonic peripheral jets. The drag for both configurations is inversely proportional to the $\mathrm{PD}$ thrust. The drag coefficient decreases from 1.5 for the no-jet case to approximately 0.87 for $C_{T}=2.5$ for the sonic jet, which corresponds to a $42 \%$ decrease. The supersonic peripheral jet produces a slightly higher drag coefficient compared to the sonic jets for all thrust coefficients, with a maximum difference of approximately $7.5 \%$. Unlike the drag, the axial force coefficient is proportional to the thrust coefficient. The axial force coefficient for $C_{T}=2.5$ is about 3.4 for the sonic jet, which is more than double the value for the no-jet case. The supersonic peripheral jet also generates slightly larger axial force coefficients than the sonic jet due to larger drag coefficients. The difference in the axial force coefficient between the two jet conditions, however, is small and does not exceed $2.5 \%$.

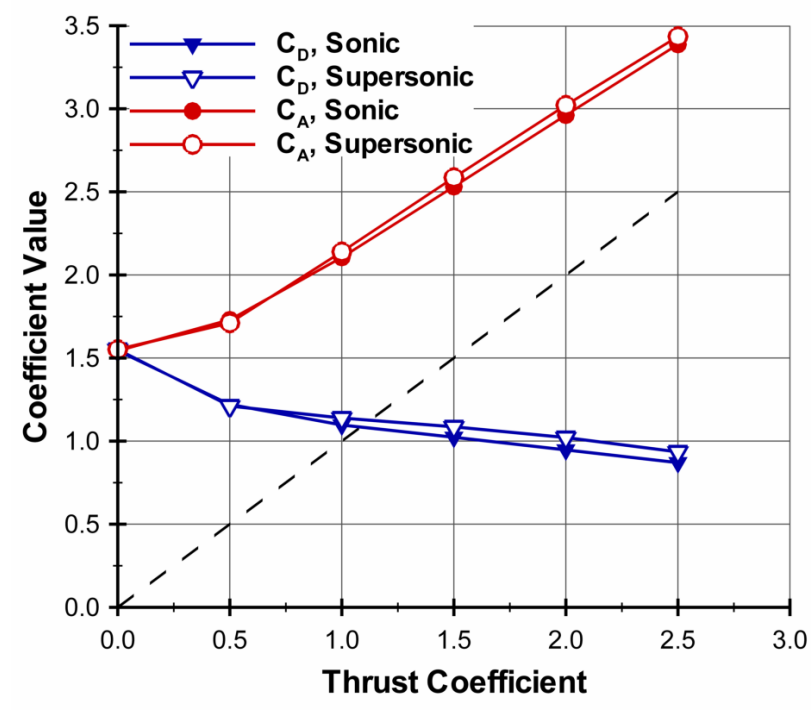

Figure 6. Drag and axial force coefficients for sonic and supersonic peripheral PD jets.

The similarities in the aeroshell deceleration performance with sonic and supersonic peripheral jets can be understood by examining the surface pressure distributions for both configurations. Figure 7 shows forebody pressure coefficient contours for the sonic and supersonic peripheral jets for various thrust coefficients. The figure also compares these results to the no-jet case. The figure shows similar surface pressure distributions for sonic and supersonic PD jets. Between two adjacent peripheral jets, the surface pressure distribution for 
both sonic and supersonic configuration resembles the no-jet case. This indicates that the peripheral jets can preserve some of the relatively high forebody pressures associated with the no-jet case. However, the figure also shows a low pressure region behind the jets (i.e. downstream of the nozzle-exit), which expands in size as the thrust coefficient increases. This low pressure region causes the decrease in drag coefficient that was observed in Figure 6.

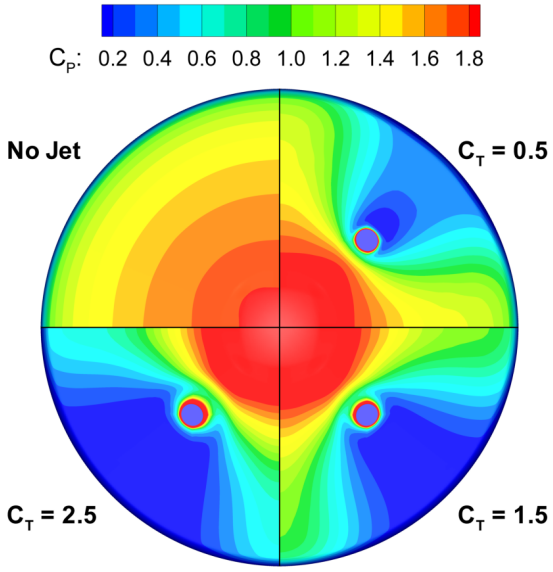

(a) Sonic

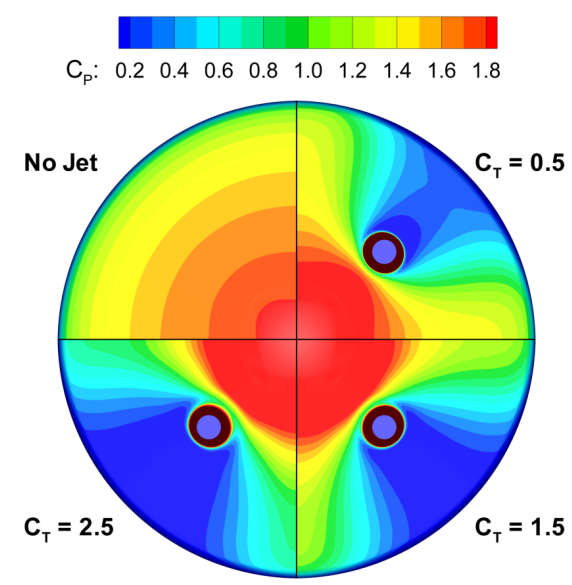

(b) Supersonic

Figure 7. Forebody pressure coefficient contours for sonic and supersonic peripheral PD jets.

The low pressure regions behind the peripheral jets, which constitute the mechanism responsible for decreasing the drag coefficient, are caused by an obstruction of the flow around the aeroshell by the PD jets. Figure 8 shows velocity streamlines for the no-jet and the sonic peripheral PD jet configurations. The blue streamlines represent particle paths originating from the freestream, while the red streamlines correspond to paths originating from the PD jet. The figure shows that the PD jet disrupts the path of the freestream particles and causes these particles to flow around the jet. This creates a wake-like region behind the PD jet, which is characterized by relatively low surface pressure. Figures $8(\mathrm{~b})$ and $8(\mathrm{c})$ also show that the level of obstruction increases with thrust coefficient since the size of the PD jet also increases. As a result, the size of the wake region (i.e. low pressure region) behind the PD jet expands and the drag coefficient decreases as the thrust coefficient increases.

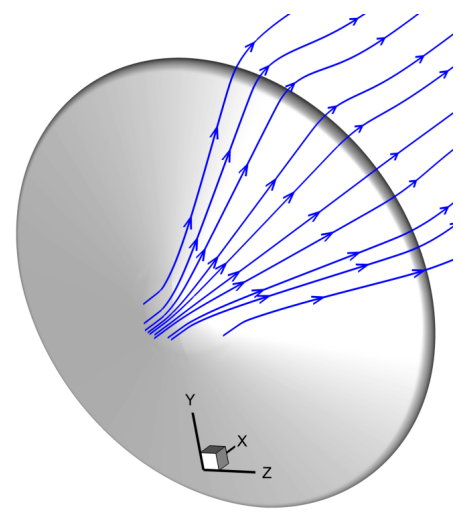

(a) No jet

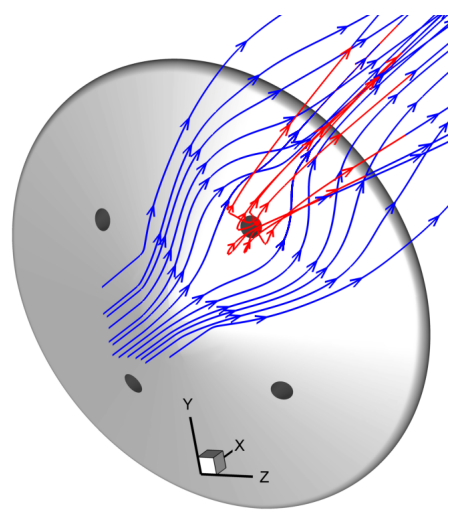

(b) $C_{T}=0.5$

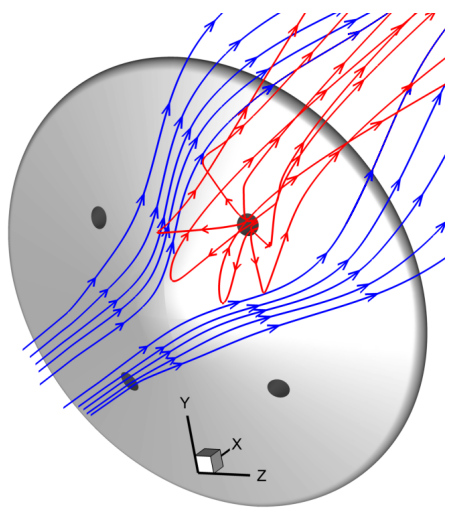

(c) $C_{T}=2.5$

Figure 8. Velocity streamlines along the aeroshell forebody for the no-jet and sonic peripheral jet configurations (blue: freestream flow; red: PD jet flow). 


\section{B. Comparisons Between Sonic Peripheral and Central PD Jets}

This section compares the flowfield and aerodynamic properties of sonic peripheral and central PD jets. Sonic central PD jets were previously analyzed in a study by Alkandry et al. ${ }^{7}$ Both configurations have the same PD nozzle dimensions and orientation. Similar to the peripheral jets, another study found that the Mach number of the PD jet for the central configuration has a small effect on the deceleration performance of Mars-entry aeroshells. ${ }^{8}$ Mach number contours and velocity streamlines for sonic peripheral and central jets are presented in Figure 9. The streamlines indicate that the peripheral jets are able to overcome the angle of the aeroshell surface at the PD nozzle-exit without generating a recirculation region, unlike the central PD jets. The peripheral jets expand to lower Mach numbers than the central jets for a given thrust coefficient because the total pressure for each peripheral jet is less than the value for the corresponding central jet in order to obtain the same overall thrust coefficient.

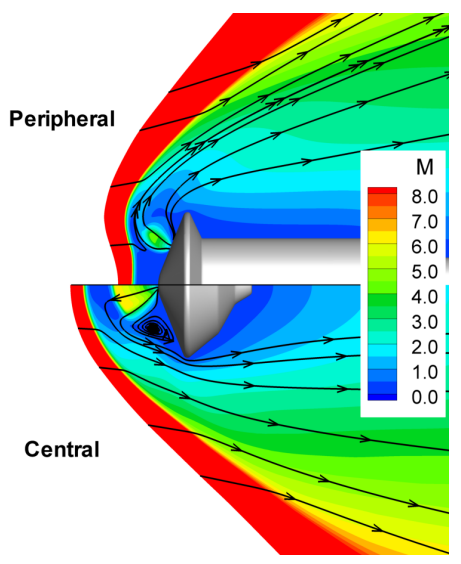

(a) $C_{T}=0.5$

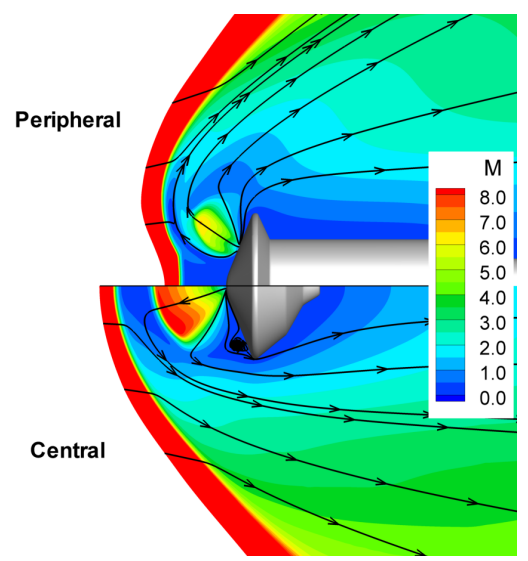

(b) $C_{T}=1.5$

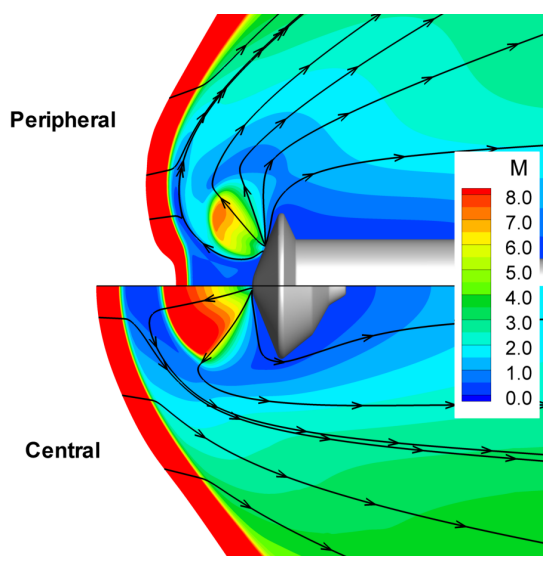

(c) $C_{T}=2.5$

Figure 9. Mach number contours for sonic peripheral (top) and central (bottom) PD jets.

Figure 10 presents the drag and axial force coefficients for the sonic peripheral and central PD configurations. The figure shows that the drag coefficient is inversely proportional to the thrust coefficient for both configurations. However, the drag coefficient for the peripheral jets is larger than for the central jets for all thrust coefficients. The difference in the drag coefficient between the peripheral and central PD jets varies from $105 \%$ for $C_{T}=0.5$ to $155 \%$ for $C_{T}=2.5$. Unlike the drag coefficient, however, the trends in the axial force coefficient for the two PD configurations are different. The axial force coefficient for the central PD jets initially decreases as the thrust coefficient increases, and then increases at $C_{T}=0.5$ mainly due to the contribution from the thrust. As a result, the axial force coefficient for the central jets does not exceed the drag coefficient for the no-jet case until a thrust coefficient of about 1.25. The axial force coefficient for the peripheral jets, however, increases for all thrust coefficients. This reveals that propulsive deceleration using peripheral jets is better than using central jets with respect to the deceleration performance of a Mars-entry aeroshell since peripheral jets are beneficial at all thrust coefficients.

The trends observed in the aerodynamic performance of aeroshells with sonic peripheral and central PD jets can be explained by examining the surface pressure distributions for the two PD configurations. Figure 11 shows forebody pressure coefficient contours for sonic peripheral and central PD jets for various thrust coefficients. The figure also compares these results with the no-jet case. As can be seen from Figure 11(b), the relatively high pressure values on the aeroshell forebody for the no-jet case are replaced by low pressure values due to the central PD jet. As discussed in a previous study, ${ }^{7}$ the low surface pressures associated with the central PD jets are caused by a "shield" that prevents mass and momentum from the freestream to reach the aeroshell surface. The study also found that the strength of the PD shield increases with thrust coefficient. As a result, less mass and momentum from the freestream reach the aersohell, which causes the pressure over the entire surface to decrease. The peripheral PD jets, on the other hand, preserve some of the high forebody pressure of the no-jet case, especially between two adjacent jets. As a result, peripheral $\mathrm{PD}$ jets produce larger drag coefficients than central jets.

The deceleration performance for sonic peripheral and central PD jets can be summarized using Figure 12. The figure shows the ratio of the axial force coefficient to an "ideal" axial force coefficient for the two 


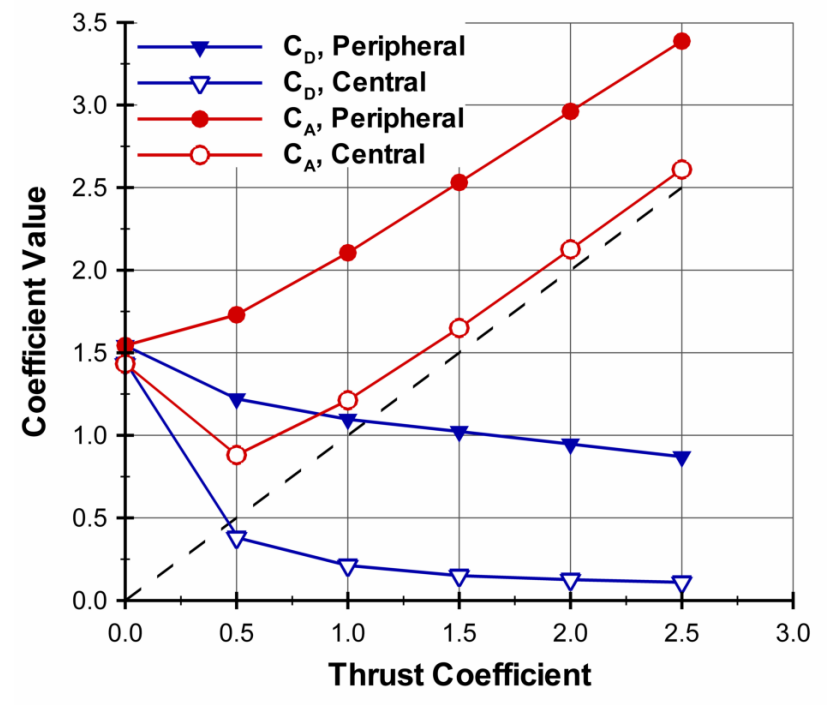

Figure 10. Drag and axial force coefficients for sonic peripheral and central PD jets.

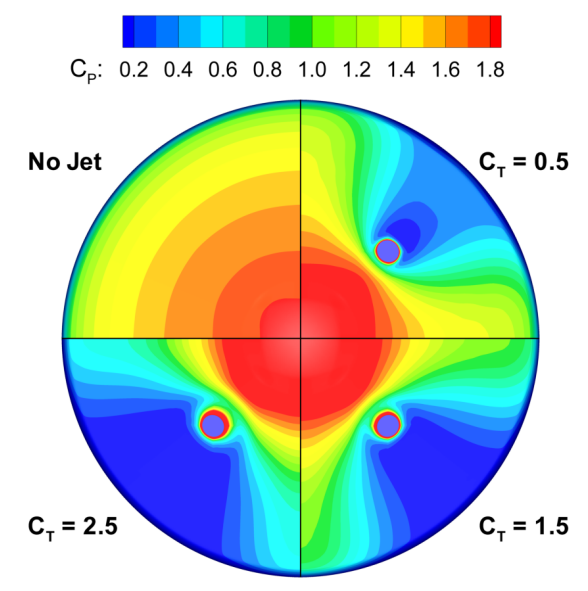

(a) Peripheral

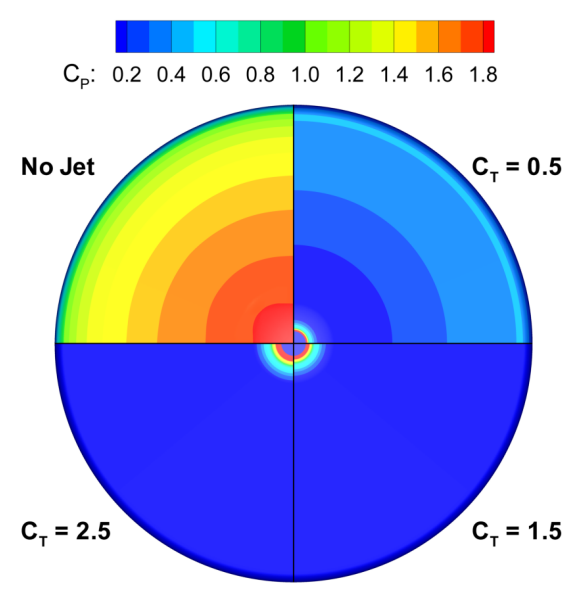

(b) Central

Figure 11. Forebody pressure coefficient contours for sonic peripheral and central PD jets. 
PD jet configurations for the thrust coefficients of interest to this study. The ideal axial force coefficient is equal to the sum of the thrust coefficient and the drag coefficient for the no-jet case. This ratio indicates no change to the axial force on the aeroshell if it is equal to 1, drag augmentation if it is greater than 1, and drag reduction if it is less than 1 . Both the peripheral and central PD jets produce axial force ratios that are less than 1 since the drag is inversely proportional to the thrust for both configurations. The axial force ratio for the peripheral jets is roughly constant at approximately 0.85 over all thrust coefficients, but the ratio for the central configuration increases from 0.45 for $C_{T}=0.5$ to 0.65 for $C_{T}=2.5$. This suggests that the deceleration performance for the peripheral jets is roughly constant at $85 \%$ of an ideal performance, while the deceleration performance for the central jets is a function of the thrust coefficient and increases from $45 \%$ to $65 \%$ for the thrust coefficients investigated in this study. This result presents another advantage of the peripheral configuration because the deceleration performance can remain roughly constant and relatively large compared to ideal conditions over a range of thrust conditions.

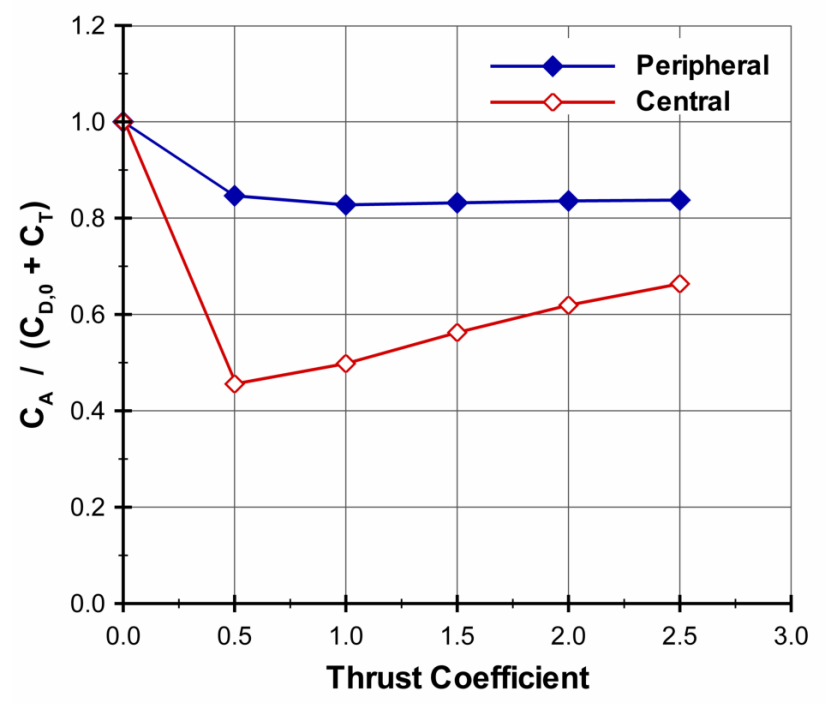

Figure 12. Deceleration performance for sonic peripheral and central PD jets.

\section{Comparisons with Experimental Data}

In order to assess the computational method, the numerical results are compared to experimental data obtained at the University of Virginia using PLIIF. ${ }^{22}$ Figures 13 and 14 show bow shock profile comparisons between the CFD results (LeMANS) and PLIIF for thrust coefficients 0.5 and 2.0. The images are PLIIF visualizations where the bright areas represent regions with relatively large $\left(\mathrm{I}_{2}\right)$ density values. The recirculation regions between the PD jet and the bow shock in the numerical results are due to the projection of the 3 -dimensional flow onto a 2-dimensional cut-plane. The figures show overall good qualitative agreement between the numerical and experimental results with respect to the bow shock profile, except for the $C_{T}=2.0$ supersonic jet case. For this case, although the results for the two methods agree well between PD jets (i.e. upstream of the aeroshell nose), the penetration depth of the PD jet predicted by LeMANS is smaller than observed in the experiment. As a result, the bow shock in the experiment is pushed farther upstream than in the numerical results. Possible causes for this disagreement are uncertainties in the experimental setup and inaccuracies in the correlation of Ashkenas and Sherman that was implemented in LeMANS to model the non-parallel streamlines in the wind tunnel, particularly far off the aeroshell centerline. 


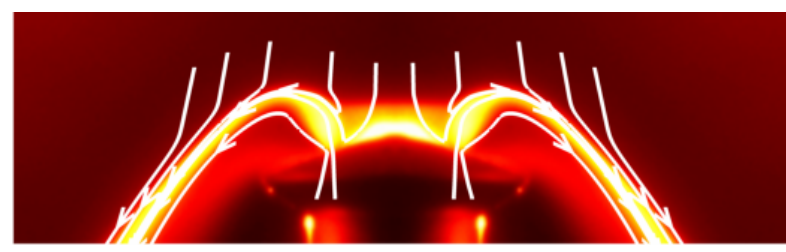

(a) $C_{T}=0.5$

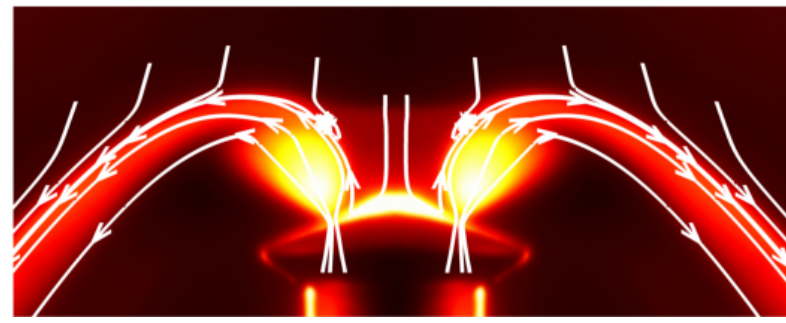

(b) $C_{T}=2.0$

Figure 13. Comparison of bow shock profile for sonic peripheral jets (images: PLIIF; line: LeMANS velocity streamlines).

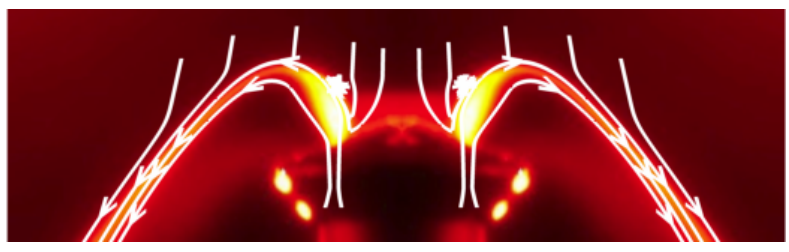

(a) $C_{T}=0.5$

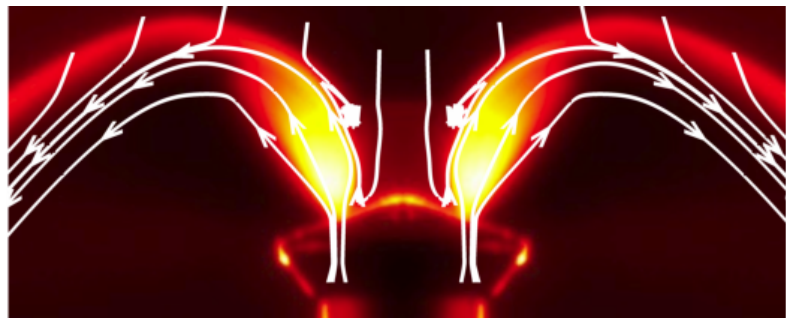

(b) $C_{T}=2.0$

Figure 14. Comparison of bow shock profile for supersonic peripheral jets (images: PLIIF; line: LeMANS velocity streamlines).

Comparisons of the bow shock standoff distance for the numerical and experimental results are presented in Figure 15. The distance is calculated along the stagnation streamline, starting at the nose of the aeroshell, and normalized by the aeroshell diameter. For the CFD results, the bow shock standoff distance corresponds to the location in the freestream where the iodine density begins to increase. The figure shows overall good agreement between the numerical and experimental results. The difference between the two sets of results decreases as the thrust coefficient increases, with a difference of approximately $30 \%$ for $C_{T}=0.5$ and about $1 \%$ for $C_{T}=2.5$ for both sonic and supersonic peripheral jets. These discrepancies are also possibly caused by uncertainties in the experimental measurements and inaccuracies in the numerical boundary conditions.

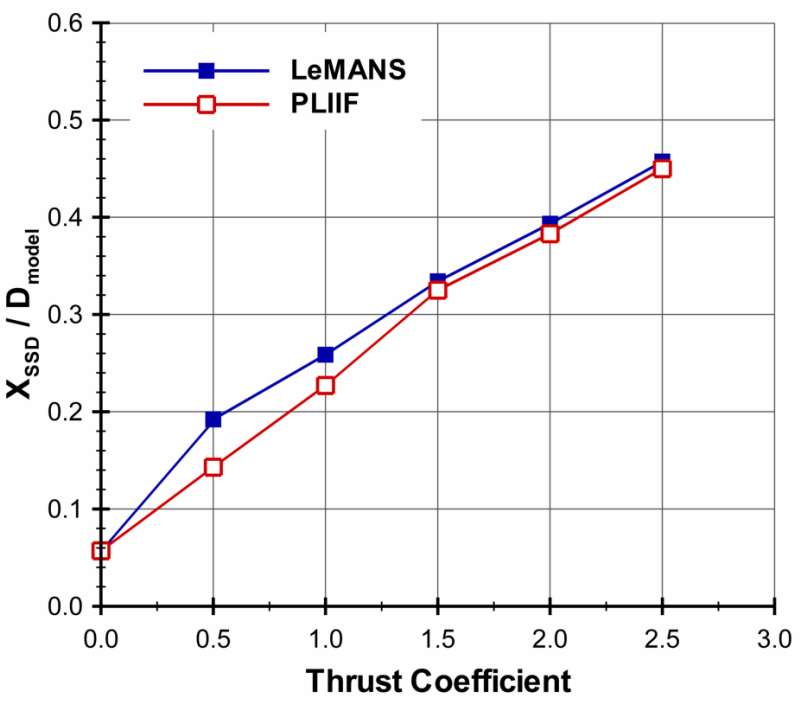

(a) Sonic jet

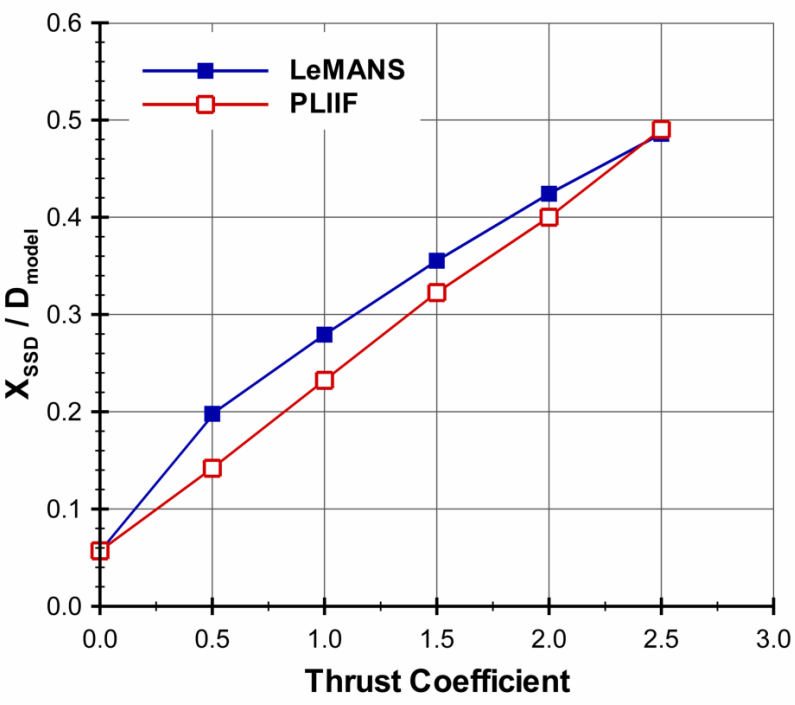

(b) Supersonic jet

Figure 15. Comparisons of bow shock standoff distance between numerical (LeMANS) and experimental (PLIIF) results for sonic and supersonic peripheral PD jets. 


\section{Conclusion}

The goal of this study was to understand the effects of peripheral PD jets on the flowfield and aerodynamic properties of Mars-entry aeroshells. Numerical simulations of an MSL-based aeroshell with 4 peripheral jets located halfway between the nose and the shoulder in Mach 12 flow of $\mathrm{I}_{2}$-seeded $\mathrm{N}_{2}$ were carried out using the CFD code LeMANS. Two different peripheral PD jet configurations, sonic and supersonic, were considered for this study in order to quantify the effects of the PD jet Mach number. The results showed that the drag coefficient for the peripheral PD jets decreased as the thrust coefficient increased because the PD jets obstructed the flow around the aeroshell which caused low pressure regions behind the jets similar to wake flows. The results also showed that the PD jet Mach number of the peripheral jets had small effects on the deceleration performance of the aeroshell, with a maximum difference less than $2.5 \%$ in the axial force coefficient between the sonic and supersonic peripheral jets. The study also compared the flowfield and aerodynamic properties of sonic peripheral and central PD jets. These comparisons revealed significant differences in the deceleration performance of the two configurations. Although the drag was inversely proportional to the thrust for both configurations, the peripheral PD jets provided 3 to 8 times more drag than the central PD jets. The reason for this was that the peripheral jets preserved some of the relatively high surface pressure associated with the no-jet case, particularly between adjacent PD jets. The axial force coefficient for the peripheral jets increased with the thrust coefficient, and, unlike the central PD jets, was always greater than the drag coefficient for the no-jet case. This is an important advantage of the peripheral jets because they improved the deceleration performance of the aeroshell over all thrust coefficients investigated in this study. Another advantage of the peripheral PD jets was that the deceleration performance remained high and roughly constant compared to an ideal performance over a range of thrust conditions. Finally, qualitative comparisons between the numerical results and experimental data obtained using PLIIF were conducted to assess the computational method. The bow shock profile and standoff distance for the two methods were in overall good agreement, with some discrepencies possibly caused by uncertainties in the experimental setup and inaccuracies in the numerical boundary conditions.

\section{Future Work}

For future work, quantitative comparisons between numerical and experimental results of flowfield and aerodynamic properties of Mars-entry aeroshells with peripheral PD jets will be carried in order to further assess the computational method. Flight conditions and enthalpies will also be considered in order to study the aerothermodynamic effects of peripheral PD jets.

\section{Acknowledgments}

The authors gratefully acknowledge funding for this work through NASA Grant NNX08AH37A. The use of supercomputers at the University of Michigan (Center for Advanced Computing) and NASA (NASA Advanced Supercomputing Division) is essential to this work and is also greatly appreciated.

\section{References}

\footnotetext{
${ }^{1}$ Braun, R. D. and Manning, R. M., "Mars Exploration Entry, Descent, and Landing Challenges," Journal of spacecraft and rockets, Vol. 44, No. 2, March-April 2007, pp. 310-323.

${ }^{2}$ Korzun, A. M., Braun, R. D., and Cruz, J. R., "Survey of Supersonic Retropropulsion Technology for Mars Entry, Descent, and Landing," Journal of Spacecraft and Rockets, Vol. 46, No. 5, September-October 2009, pp. 929-937.

${ }^{3}$ Edquist, K. T., Dyakonov, A. A., Korzun, A. M., Shidner, J. D., Studak, J. W., Tigges, M. A., Kipp, D. M., Prakash, R., Trumble, K. A., and Dupzyk, I. C., "Development of Supersonic Retro-Propulsion for Future Mars Entry, Descent, and Landing Systems," AIAA Paper 2010-5046, June 2010.

${ }^{4}$ Berry, S. A., Laws, C. T., Kleb, W. L., Rhode, M. N., Spells, C., Mccrea, A. C., Trumble, K. A., Schauerhamer, D. G., and Oberkampf, W. L., "Supersonic Retro-Propulsion Experimental Design for Computational Fluid Dynamics Model Validation," Aerospace Conference, 2011 IEEE, March 2011.

${ }^{5}$ Trumble, K. A., Schauerhamer, D., Kleb, W. L., Carlson, J. R., and Edquist, K. T., "Analysis of Navier-Stokes codes applied to Supersonic Retro-Propulsion wind tunnel test," Aerospace Conference, 2011 IEEE, March 2011.

${ }^{6}$ Dwyer-Cianciolo, A. M., Davis, J. L., Komar, D. R., Munk, M. M., Samareh, J. A., Powell, R. W., Shidner, J. D., Stanley, D. O., Wilhite, A. W., Kinney, D. J., McGuire, M. K., Arnold, J. O., Howard, A. R., Sostaric, R. R., Studak, J. W., Zumwalt, C. H., Llama, E. G., Casoliva, J., Ivanov, M. C., Clark, I., and Sengupta, A., "Entry, Descent and Landing Systems Analysis Study: Phase 1 Report," NASA TM-2010-0000002009, July 2010.
} 
${ }^{7}$ Alkandry, H., Boyd, I. D., Reed, E. M., Codoni, J. R., and McDaniel, J. C., "Interactions of Single-Nozzle Sonic Propulsive Deceleration Jets on Mars Entry Aeroshells," AIAA Paper 2010-4888, June 2010.

${ }^{8}$ Alkandry, H., Boyd, I. D., Reed, E. M., Codoni, J. R., and McDaniel, J. C., "Interactions of Single-Nozzle Supersonic Propulsive Deceleration Jets on Mars Entry Aeroshells," AIAA-2011-138, January 2011.

${ }^{9}$ McDaniel, J. C., Glass, C. E., Staack, D., and Miller, C. G., "Experimental and Computational Comparisons of an Underexpanded Jet Flowfield," AIAA Paper 2002-0305, January 2002.

${ }^{10}$ Cecil, D. E. and McDaniel, J. C., "Planar Laser-Induced Iodine Fluorescence Measurements in Rarefied Hypersonic Flow," Rarefied Gas Dynamics: 24th International Symposium, Toronto, Canada, 2005, pp. 1325-1350.

${ }^{11}$ Reed, E. M., Codoni, J., McDaniel, J. C., Alkandry, H., and Boyd, I. D., "Investigation of the Interactions of Reaction Control Systems with Mars Science Laboratory Aeroshell," AIAA Paper 2010-1558, January 2010.

${ }^{12}$ Scalabrin, L. C. and Boyd, I. D., "Development of an Unstructured Navier-Stokes Solver for Hypersonic Nonequilibrium Aerothermodynamics," AIAA Paper 2005-5203, June 2005.

${ }^{13}$ Scalabrin, L. C. and Boyd, I. D., "Numerical Simulation of Weakly Ionized Hypersonic Flow for Reentry Configurations," AIAA Paper 2006-3773, June 2006.

${ }^{14}$ Holman, T. D. and Boyd, I. D., "Effects of Continuum Breakdown on the Surface Properties of a Hypersonic Sphere," Journal of Thermophysics and Heat Transfer, Vol. 23, No. 4, October-December 2009, pp. 660-673.

${ }^{15}$ Wilke, C. R., "A Viscosity Equation for Gas Mixtures," Journal of Chemical Physics, Vol. 18 No. 4, 1950, pp. 517-519.

${ }^{16}$ Blottner, F. G., Johnson, M., and Ellis, M., "Chemically Reacting Viscous Flow Program for Multi-Component Gas Mixtures," Tech. rep., SC-RR-70-754, Sandia Laboratories, Albuquerque, New Mexico, 1971.

${ }^{17}$ Vincenti, W. G. and Kruger, C. H., Introduction to Physical Gas Dynamics, Krieger Publishing Company, 2002.

${ }^{18}$ Karypis, G. and Kumar, V., "METIS: A Software Package for Partitioning Unstructured Graphs, Partitioning Meshes, and Computing Fill-Reducing Orderings of Sparse Matrices," University of Minnesota, 1998.

${ }^{19}$ Ashkenas, H. and Sherman, F. S., "The Structure and Utilization of Supersonic Free Jets in Low Density Wind Tunnels," Proceedings of the 4th International Symposium on Rarefied Gas Dynamics, Academic Press, 1966, pp. 84-105.

${ }^{20}$ Alkandry, H., Boyd, I. D., Reed, E. M., and McDaniel, J. C., "Numerical Study of Hypersonic Wind Tunnel Experiments for Mars Entry Aeroshells," AIAA Paper 2009-3918, June 2009.

${ }^{21}$ Pope, S. B., Turbulent Flows, Cambridge University Press, 2000.

${ }^{22}$ Codoni, J. R., Reed, E. M., McDaniel, J. C., Alkandry, H., and Boyd, I. D., "Investigations of Peripheral 4-jet Sonic and Supersonic Propulsive Deceleration Jets on a Mars Science Laboratory Aeroshell," AIAA 2011-1036, January 2011. 